Produto \& Produção, vol. 7, n. 3, p. 51-62, out. 2004

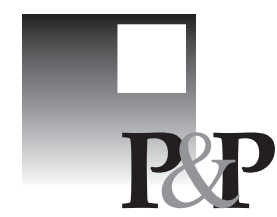

\title{
Análise dos instrumentos utilizados para a avaliação do risco da ocorrência dos D.O.R.T./L.E.R.
}

\author{
Luis Ulisses Signori \\ Rua General Felipe Portinho, 1502 \\ 98050005 - Cruz Alta, RS \\ signori@laguna.com.br
}

Lia Buarque de Macedo Guimarães, $P h D$.

liabmg@ppgep.ufrgs.br

Renan Maximiliano Fernandes Sampedro, $D r$.

Avenida Itaimbé, 221 apt. 403

97.050-331 Santa Maria, RS

renansam@terra.com.brou sampedro@main.unicruz.tche.br

\begin{abstract}
O presente estudo buscou analizar a aplicabilidade e a fidedignidade de 9 instrumentos utilizados pelos ergonomistas na avaliação e classificação dos riscos de ocorrência dos D.O.R.T./L.E.R.. Os instrumentos selecionados foram: os Chek Lists de Lifshitz e Armstrong (1986 apud OLIVEIRA, 1998), Keyserling et al. (1993) e Couto (1998); os critérios semiquantitativos de Karu, Kansi e Kuorinka (1977) conhecido como OWAS, o protocolo de Rodgers (1992), o de McAtamney e Corlett (1993) denominado RULA e o protocolo de Malchaire (1998); e os critérios quantitativos de Moore e Garg (1995) e a minuta da International Ergonomics Association desenvolvida por Colombini et al. (1999). Dezoito acadêmicos do $8^{\circ}$ período do Curso de Fisioterapia da UNICRUZ utilizaram os nove instrumentos na avaliação de dois postos de trabalho da indústria eletroeletrônica. O critério de seleção dos postos foi a repetitividade da tarefa, sendo um posto com ciclo repetitivo (ciclo < que 30s) e outro não. Os resultados apresentaram uma grande variabilidade intra e intergrupo, o que representa uma baixa fidedignidade nos instrumentos de avaliação, podendo um mesmo posto de trabalho apresentar uma classificação de baixo, moderado ou alto risco de ocorrência dos D.O.R.T./L.E.R., dependendo do protocolo utilizado e do analista. Os instrumentos que apresentaram menor variação de resultados foram o OWAS (KAHRU et al., 1977) na análise do segmento dos membros inferiores, I.E.A. Colombini et al. (1999) na análise dos membros superiores e RULA (1993) na análise geral do posto.
\end{abstract}

Palavras-chave: instrumentos de avaliação de postura; protocolos; posto de trabalho; D.O.R.T./L.E.R.

The study analyses the applicability and reliability of nine different instruments used by ergonomists for WMSDS/ CTDs's risk evaluation. The selected instruments were: the Check Lists of Lifshitz and Armstrong* (1986 apud OLIVEIRA, 1998), Keyserling et al. (1993) and Couto (1998); the semiquantitative criterion of: Karu, Kansi and Kuorinka (1977) well-known as OWAS, Rodgers (1992), Malchaire (1998), and McAtamney and Corlett (1993), named RULA; and the quantitative criterion of: Moore \& Garg (1995), and the memorandum of the International Ergonomics Association written by Colombini et al. (1995). The instruments were used by eighteen physiotherapists for the analisys of two different assembly workplaces at an electronic industry: one wokplace was defined as repetitive (cycle < than 30s) and the other one, not-repetitive. The results showed a great variance intra and intergroup, which represents a low reliability of the instruments used for evaluation: a same workplace was classified as low, moderate or high risk for the occurrence of WMSDS/CTDs, depending on the analyst and the instrument used. The instruments with less result variability were: OWAS (KAHRU et al., 1977) on the analysis of the lower limbs, I.E.A. Colombini et al. (1999) on the analysis of the upper limbs and RULA (1993) on the general evalution of the workplace.

Keywords: instruments for posture analysis; workstation; W.M.S.D.S/C.T.D.

\footnotetext{
* LIFSHITZ, Y.; ARMSTRONG, J. (1986) A design checklist for control and prediction of cumulative trauma disorder in intensive manual jobs. THE HUMAN FACTORS SOCIETY ANNUAL MEETING, 30. Proceedings... pp. 837-941.
} 


\section{Introdução}

Os Distúrbios Osteomusculares Relacionados ao Trabalho (D.O.R.T./L.E.R.) tornaram-se, em todo o mundo, a doença dos anos noventa, principalmente devido à informatização do trabalho que reduz a variedade de ações do trabalhador e induz a posturas mais estáticas (DIMON, 1997). Nos países do terceiro mundo, este problema é agravado pela realidade socioeconômica, pois as empresas tendem a não fornecer condições favoráveis de trabalho, quer ambientais, quer de posto, quer organizacionais. Sem um suporte oficial de órgãos governamentais de apoio ao trabalhador, sindicatos etc., e sob o risco da demissão, o trabalhador fica inibido de reivindicar condições ambientais mais seguras, postos mais adequados, ritmo e jornada de trabalho mais condizentes com suas capacidades, o que reduziria a incidência dos acidentes de trabalho e das doenças ocupacionais.

No Brasil, os Distúrbios Osteomusculares Relacionados ao Trabalho foram reconhecidos inicialmente sob a denominação de Lesões por Esforços Repetitivos (L.E.R.), a partir da portaria 4062 do INSS de 06/07/87 (BRASIL, 1987). L.E.R é tradução do termo Repetition Strain Injuries (R.S.I.) utilizado inicialmente na Austrália e definido por Browne, em 1984 (COUTO et al., 1998). No entanto, o termo L.E.R é inapropriado, pois a repetitividade é apenas um (e nem sempre está presente) de um conjunto de fatores biomecânicos causais. A aceitação de que a minimização da repetitividade, isoladamente, reduziria o aparecimento de novos casos de D.O.R.Ts, é simplificar um quadro que, na realidade, é mais complexo. Desta forma, a Real Sociedade Australiana de Medicina recomendou, oficialmente, em 1986, que o termo "Repetition Strain Injuries" fosse abandonado e se passasse a usar o termo "Syndrome Regional Painful of Occupational Origin", ou seja, "Síndrome Dolorosa Regional de Origem Ocupacional" (OLIVEIRA et al., 1998). Com base nesta idéia, no Brasil, o Decreto no 2172 de 05/03/ 97 (BRASIL, 1998) impõe a adoção do termo Distúrbios Osteomusculares Relacionados ao Trabalho (D.O.R.T.) em substituição a outras denominações, tais como: Lesões por Traumas Cumulativos (L.T.C.), Lesões por Esforços Repetitivos (L.E.R.), Doença Cervicobraquial Ocupacional (D.C.O.), Síndrome de Sobrecarga Ocupacional (S.S.O.) etc. Isto se faz necessário para eliminar o nexo causal embutido nestes termos que, nas próprias denominações, apontam causas definidas (como por exemplo, "cumulativa" nas L.T.C. e "repetitivas" nas L.E.R.) e os efeitos (como por exemplo: "lesões" nas L.T.C. e L.E.R.). A terminologia D.O.R.T destaca a expressão "distúrbio", ao invés de "lesões", pois corresponde ao que se percebe, na prática: ocorrerem distúrbios numa fase precoce (fadiga, sensação de peso nos membros etc.) e lesões mais tardiamente (deposição de tecido cicatricial) (COUTO et al., 1998). Apesar do decreto, ainda é comum a generalização de distúrbios pelo termo L.E.R e, portanto, com o objetivo de manter coerência com a literatura brasileira e a praxis atual, o termo D.O.R.T./L.E.R. será utilizado em todo este trabalho.

Apesar de não haver dados precisos no Brasil, suspeita-se que, assim como nos E.U.A. (LUDUVIG, 1998; OLIVEIRA et al., 1998), os D.O.R.T./L.E.R. sejam responsáveis por $65 \%$ das licenças médicas solicitadas pelos trabalhadores brasileiros. O National Institute of Occupacional Safety and Health (NIOSH) previu que no ano 2000, $50 \%$ da força de trabalho americana sofreria as conseqüências destes distúrbios (D.O.R.T./L.E.R) (NIOSH, 1998).

Os fatores de risco dos D.O.R.T./L.E.R. são de natureza: biomecânica (postura, força, repetitividade, compressões mecânicas e vibrações); organizacional (ausência de pausas, horas-extras, duração da jornada de trabalho, fragmentação da tarefa etc.); psicossocial (a expressão das percepções subjetivas que o trabalhador tem dos fatores da organização do trabalho); ambiental (temperaturas abaixo de $21^{\circ} \mathrm{C}$ ) e individual (anticoncepcionais orais, menopausa, esterectomia com ovarectomia; patologias associadas, tais como: mixedema, amiloidose, hiper-tiroidismo, gota, artrite reumatóide, diabetes; lesões ou traumas antigos; e às atividades extraprofissio-nais, sejam elas domésticas ou esportivas). Os D.O.R.T./L.E.R. surgem quando a intensidade dos fatores de riscos ultrapassa a capacidade de recuperação dos tecidos. Portanto, a detecção, a classificação e a avaliação da intensidade dos fatores de risco no posto de trabalho servem de base para o diagnóstico e para as propostas de solução dos problemas em uma intervenção ergonômica que busca a prevenção dos D.O.R.T./L.E.R..

O objetivo deste trabalho foi o de realizar uma análise de instrumentos utilizados em situação de campo, para a detecção e classificação do risco da ocorrência dos D.O.R.T./L.E.R. Com base na revisão da literatura sobre os fatores predisponentes e sobre os instrumentos de avaliação do risco da ocorrência dos D.O.R.T./L.E.R. mais utilizados na atualidade, foi realizada a análise de dois postos de trabalho com a aplicação de nove instrumentos considerados de fácil aplicação em campo, e analisados os resultados.

O interesse principal na avaliação dos nove instrumentos selecionados era identificar se eles apresentam pouca variação dos resultados, sendo, portanto, fidedignos. A fidedignidade dos resultados destes instrumentos é de fundamental importância para o ergonomista realizar a triagem dos postos de traba- 
lho nas empresas, identificar os problemas, implementar as melhorias e após, ter uma base para comparação de resultados e comprovação dos benefícios da intervenção ergonômica.

\section{Materiais e métodos}

\section{Seleção dos postos}

A pesquisa compreendeu a avaliação de dois postos de trabalho da empresa Asea Brown Boveri (ABB), indústria eletroeletrônica, fabricante de medidores elétricos, situada na cidade de Cachoeirinha - RS, a qual mantinha convênio com o PPGEP/UFRGS para projetos de extensão em Ergonomia.

O critério de seleção dos postos de trabalho foi a repetitividade, de acordo com Silverstein et al. (1986), que estabelecem, como repetitivo, um trabalho com ciclo inferior a 30 segundos. Selecionou-se dois postos de trabalho: o posto 1, considerado repetitivo por este critério (ciclo de trabalho $=$ $27,6 \mathrm{~s} \pm 7,16$ s) e o posto 2 , considerado não repetitivo (ciclo de trabalho $=141,5 \mathrm{~s} \pm 3 \mathrm{~s}$ ). O ambiente de trabalho, de ambos os postos, é climatizado, sendo observado, no dia do levantamento dos dados (21 de julho de 1999): a temperatura ambiente: temperatura de bulbo seco $(\mathrm{TBS})=24,5^{\circ} \mathrm{C}$; temperatura de bulbo úmido $(\mathrm{TBU})=20^{\circ} \mathrm{C}$; velocidade do ar 0 $\mathrm{m} / \mathrm{s}$; umidade relativa do $\mathrm{ar}=55 \%$.

O Posto 1 é o de pré-calibração do medidor monofásico M8C. Neste posto, a tarefa compreende as atividades de: pegar o medidor da esteira; posicionar o medidor no calibrador; desmagnetizar o freio; realizar as análises visuais de qualidade de montagem; ajustar a carga nominal; ajustar a carga indutiva; ajustar carga pequena e; recolocar o medidor na esteira. O Posto 2 é o Posto de montagem inicial do medidor polifásico TELFT1914Padrão. Neste posto, a tarefa compreende: pegar o medidor da esteira; posicionar o medidor; realizar seis ligações elétricas; colocar o medidor no bloco; fixar o bloco no medidor com dois parafusos; colocar a máscara; fixar dezesseis parafusos, testar as seis ligações elétricas; realizar a limpeza com o jato de ar; e colocar o medidor no próximo posto de trabalho.

\section{Sujeitos sob observação}

A população estudada compreende os trabalhadores do setor de montagem dos medidores que atuam no seu posto de trabalho na postura preponderantemente estática, (sentado, em pé, ou de pé/sentado), sem rodízio e sem deslocamentos fora do setor.

Foram observados dois trabalhadores em cada posto, executando a tarefa real, que foi registrada em vídeo. Todos os trabalhadores foram informados das filmagens e dos objetivos do estudo, tendo concordado com a sua participação voluntária no estudo.

\section{Procedimentos de Coleta de dados}

O trabalho nos postos foi filmado garantindo-se cinco ciclos de trabalho para cada posto. Foram tentadas filmagens durante todo o ciclo em todas as direções possíveis, ou seja: nas posições lateral esquerda, lateral direita, anterior, posterior e específica para as mãos. No entanto, como a disposição dos postos de trabalho não permitia as filmagens em todas estas posições (pois não havia espaço livre para a colocação da câmera de vídeo) as filmagens não puderam ser iguais nos dois postos.

No Posto 1 (Posto de Pré-calibração), foram filmados: dois ciclos, estando a filmadora posicionada a 2,5 metros (m) à direita dos trabalhadores; dois ciclos com a câmera posicionada a $2,5 \mathrm{~m}$ posterior aos trabalhadores e; um ciclo específico filmando as mãos dos trabalhadores, com a câmera a $1 \mathrm{~m}$.

No Posto 2 (Posto de Montagem Inicial), foram obtidas as imagens a 2,5m para: um ciclo lateral direito; um ciclo lateral esquerdo; um ciclo posterior; um ciclo anterior. A $1 \mathrm{~m}$ foi filmado um ciclo específico para as mãos.

Anteriormente às filmagens, foram coletados os dados da percepção subjetiva de esforço de cada trabalhador, por meio da escala modificada de $\operatorname{Borg}(0$ a 10$)$

(AMERICANCOLLEGEOFSPORTS MEDICINE, 1996).

\section{Procedimentos de Análise de dados}

A avaliação do risco da ocorrência dos D.O.R.T./ L.E.R. foi verificada com base nos filmes, segundo os nove instrumentos selecionados, por dezoito acadêmicos legalmente matriculados no $8^{\circ}$ semestre do Curso de Fisioterapia da Universidade de Cruz Alta (UNICRUZ) no Rio Grande do Sul.

Além dos filmes dos dois postos de trabalho, foi oferecido aos acadêmicos um relatório com a descrição das tarefas, avaliação ambiental dos postos e os resultados da escala de Borg. Para garantir que os acadêmicos estavam aptos a utilizar todos os instrumentos, eles fizeram um curso de 20 horas/aula para instrumentação e o uso dos protocolos.

O conteúdo programático desenvolvido no curso foi dividido em duas partes:

- a primeira parte, direcionada à revisão teórica dos conceitos e definição de termos dos D.O.R.T./ L.E.R., aspectos epidemiológicos, localização e classificação dos principais distúrbios de acordo 
com o Código Internacional de Doenças, $10^{\mathrm{a}}$. Ed. (C.I.D./10), análise e os principais critérios de avaliação dos fatores de risco.

- a segunda parte compreendeu a aprendizagem dos nove instrumentos de análise. Três Check Lists: de Lifshitz e Armstrong* (1986 apud OLIVEIRA, 1998), Keyserling et al. (1993) e Couto (1998); quatro protocolos semiquantitativos, o de Karu, Kansi e Kuorinka (1977) conhecido como OWAS, o protocolo de Rodgers (1992), o de Mcatamney e Corlett (1993) denominado RULA e o protocolo de Malchaire (1998); e dois instrumentos quantitativos: o de Moore e Garg (1995) e a minuta da International Ergonomics Association (conhecido como I.E.A), desenvolvida por Colombini et al. (1999).

Os instrumentos foram empregados na sua íntegra, exceto o método de Malchaire (1998) e a minuta da I.E.A. (COLOMBINI et al., 1999). O método de Malchaire (1998) compreende quatro etapas, mas nesta pesquisa coletaram-se os dados apenas da etapa 2. A minuta da I.E.A. foi o último instrumento a ser empregado, sendo utilizados os critérios para a avaliação dos fatores de risco da ocorrência dos D.O.R.T./L.E.R., descritos em seu artigo original de 1995 descrito em Couto (1998). Salienta-se que este protocolo sofreu alterações em 1999 (COLOMBINI et al., 1999), mas as fórmulas para calcular o LATARE (Limite de Ações Técnicas Recomendadas) e o IEMS (Índice de Exposição dos Membros Superiores) permanecem os mesmos.

O Posto 2 (Posto de Montagem Inicial) foi avaliado, pelos nove instrumentos, em sala de aula durante o curso. O Posto 1 foi avaliado pelos acadêmicos, individualmente, em casa, sendo que cada acadêmico recebeu uma fita de vídeo do Posto 1 . Tendo em vista não ter ocorrido a interferência do professor e colegas, os resultados do Posto 1 mostraram-se mais fidedignos e, portanto, foram os utilizados na discussão deste estudo.

\section{Resultados e discussão}

A Figura 1 apresenta as médias e os desviospadrão dos resultados de cada um dos instrumentos de avaliação no Posto 1 . A Tabela 1 refere-se aos resultados da análise do Posto de Précalibração do Medidor Monofásico M8C. Os instrumentos foram padronizados de acordo com a classificação do risco: baixo (1), moderado (2) e alto (3) de ocorrência dos D.O.R.T./L.E.R. Nos protocolos que apresentavam mais do que estas três faixas de risco, as faixas foram agrupadas e enquadradas nestas três. Os dados foram normalizados com a média individual de cada avaliador para posterior aplicação das demais ferramentas estatísticas. Para o mesmo segmento corporal, foi utilizado o teste " $\mathrm{t}$ " de Student para duas variáveis e a ANOVA (Análise de Variância) de Fator Simples para mais de duas variáveis. Utilizou-se para o teste Post Hoc de Newman-Keuls para análise de diferenças, sendo adotado o nível de significância $\mathrm{p} \leq 0,05$.

A análise de variância mostra que existe uma variação significativa dos resultados dos nove instrumentos de avaliação do risco da ocorrência dos D.O.R.T./L.E.R. para o Posto de trabalho 1. A Tabela 2 apresenta o teste de análise Post Hoc das diferenças apontadas pela análise de variância dos diferentes instrumentos de avaliação dos riscos dos D.O.R.T./L.E.R. Pelo teste Post Hoc de NewmanKeuls, pode-se agrupar os resultados em três grupos distintos que expressam a classificação de alto $\left(^{\mathrm{A}}\right)$, moderado $\left({ }^{\mathrm{B}}\right)$ e baixo $\left({ }^{\mathrm{C}}\right)$ risco da ocorrência dos D.O.R.T./L.E.R. relacionados ao Posto de Pré Calibração do medidor Monofásico M8C.

Tabela 1 - Análise de variância da classificação do risco da ocorrência dos D.O.R.T./L.E.R. propostos pelos 9 instrumentos utilizados.

\begin{tabular}{lccccc}
\hline Fonte da variação & SQ & Gl & MQ & F & valor-P \\
\hline Entre grupos & 163,6574 & 23 & 7,1155 & $* 24,2751$ & $6,28 \mathrm{E}-62$ \\
\hline Dentro dos grupos & 119,5929 & 408 & 0,2953 & & \\
\hline Total & 283,2503 & 431 & & & \\
\hline
\end{tabular}

* Significante para $\mathrm{p} \leq 0,05$

\footnotetext{
* LIFSHITZ, Y.; ARMSTRONG, J. (1986) A design checklist for control and prediction of cumulative trauma disorder in intensive manual
} jobs. THE HUMAN FACTORS SOCIETY ANNUAL MEETING, 30. Proceedings... pp. 837-941. 


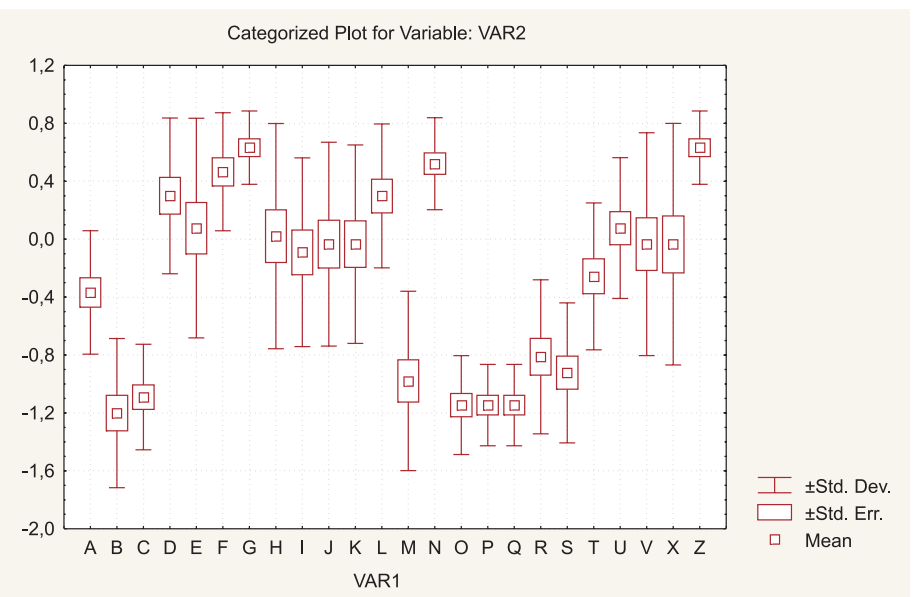

Figura 1 - Distribuição da classificação do risco da ocorrência dos D.O.R.T./L.E.R. no Posto 1 segundo os 9 instrumentos de avaliação utilizados.

Tabela 2 - Análise Post Hoc, teste de Newman-Keuls, para avaliar as diferenças na classificação o risco da ocorrência dos D.O.R.T./L.E.R. segundo 9 instrumentos utilizados utilizados.

\begin{tabular}{|c|c|c|c|c|}
\hline PROTOCOLOS & LETRA & SEGMENTO & MÉDIA & DESV. PAD. \\
\hline Lifshitz \& Armstrong (1986) & A & M.S. & $-0,3678^{\text {в }}$ & $\pm 0,4263$ \\
\hline \multirow{2}{*}{ Keyserling et al. (1993) } & $\mathrm{B}$ & M.S.Esq. & $-1,2012^{\mathrm{C}}$ & $\pm 0,5151$ \\
\hline & $\mathrm{C}$ & M.S.Dir. & $-1,0900^{\mathrm{C}}$ & $\pm 0,3643$ \\
\hline Couto (1998) & $\mathrm{D}$ & M.S. & $0,2987 \mathrm{AB}$ & $\pm 0,5377$ \\
\hline \multirow{3}{*}{ OWAS (1977) } & $\mathrm{E}$ & Coluna Dorsal & $0,0765 \mathrm{AB}$ & $\pm 0,7583$ \\
\hline & $\mathrm{F}$ & Braço (M.S.) & $0,4654^{\mathrm{A}}$ & $\pm 0,4075$ \\
\hline & G & Pernas (M.I.) & $0,6321^{\mathrm{A}}$ & $\pm 0,2532$ \\
\hline \multirow{6}{*}{ Rodgers (1992) } & $\mathrm{H}$ & Coluna cervical & $0,0210 \mathrm{AB}$ & $\pm 0,7775$ \\
\hline & I & Ombro & $-0,0900 \mathrm{AB}$ & $\pm 0,6515$ \\
\hline & $\mathrm{J}$ & Coluna dorsal & $-0,0345 \mathrm{AB}$ & $\pm 0,7038$ \\
\hline & $\mathrm{K}$ & Cotovelos & $-0,0345 \mathrm{AB}$ & $\pm 0,6853$ \\
\hline & $\mathrm{L}$ & Punhos/mãos & $0,2987 \mathrm{AB}$ & $\pm 0,4968$ \\
\hline & M & M.I. & $-0,9789$ вС & $\pm 0,6192$ \\
\hline RULA (1993) & $\mathrm{N}$ & Geral & $0,5210^{\mathrm{A}}$ & $\pm 0,3177$ \\
\hline \multirow{7}{*}{ Malchaire (1998) } & $\mathrm{O}$ & Coluna cervical & $-1,1456^{\mathrm{C}}$ & $\pm 0,3413$ \\
\hline & $\mathrm{P}$ & Ombro Dir. & $-1,1456^{\mathrm{C}}$ & $\pm 0,2808$ \\
\hline & Q & Ombro Esq. & $-1,1456^{\mathrm{C}}$ & $\pm 0,2808$ \\
\hline & $\mathrm{R}$ & Cotovelo Dir. & $-0,8123^{\text {ВС }}$ & $\pm 0,5316$ \\
\hline & $\mathrm{S}$ & Cotovelo Esq. & $-0,9234^{\mathrm{BC}}$ & $\pm 0,4834$ \\
\hline & $\mathrm{T}$ & Punho/mão Esq & $-0,2567^{\text {в }}$ & $\pm 0,5073$ \\
\hline & $\mathrm{U}$ & Punho/mão Dir & $0,0765^{\mathrm{AB}}$ & $\pm 0,4861$ \\
\hline \multirow{2}{*}{ Moore \& Garg (1995) } & $\mathrm{V}$ & Extrem. M.S. Dir. & $-0,0345 \mathrm{AB}$ & $\pm 0,7694$ \\
\hline & $\mathrm{X}$ & Extrem. M.S. Esq. & $-0,0345 \mathrm{AB}$ & $\pm 0,8340$ \\
\hline I.E.A. (1995) & $\mathrm{Z}$ & M.S. & $0,6321^{\mathrm{A}}$ & $\pm 0,2532$ \\
\hline Média & & & $-0,2613$ & \\
\hline Desvio Padrão & & & $\pm 0,6286$ & \\
\hline
\end{tabular}

Letras sobrescritas iguais não existem diferença significante entre si, para $p \leq 0,05$

${ }^{\mathrm{A}}=$ alto $(0,252$ a 0,900$),{ }^{\mathrm{B}}=\operatorname{moderado}(0,251$ a $-0,793) \mathrm{e}^{\mathrm{C}}=$ baixo $(-0,794$ a $-1,800)$ 
$\mathrm{Na}$ análise dos nove instrumentos, verifica-se uma média $-0,2613$, um desvio padrão de $\pm 0,6286$, caracterizando um risco geral entre moderado a alto para a ocorrência dos D.O.R.T/L.E.R. no Posto de Précalibração do Medidor Monofásico M8C.

Pôde-se discutir apenas os resultados dos instrumentos que avaliam o mesmo segmento corporal, de acordo com os estudos de fisiologia articular propostos por Kapandji (1990). Conforme a Tabela 3, observou-se não existir diferença significativa entre métodos que avaliam os segmentos da coluna vertebral dorsal (C.V.D.) e punhos e mãos (P/M). No entanto, apresentaram diferenças estatísticas significantes os métodos que avaliam os segmentos da coluna vertebral cervical (C.V.C.), membros superiores (M.S.), membros inferiores (M.I.), ombros $(\mathrm{O})$ e cotovelos $(\mathrm{C})$.

Tabela 3 - Análise do teste Post Hoc de Newman-Keuls para avaliar as diferenças na classificação o risco da ocorrência dos D.O.R.T./L.E.R. propostos pelos 9 instrumentos analisados por segmentos articular

\begin{tabular}{|c|c|c|c|}
\hline PROTOCOLOS & LETRA & SEGMENTO & $\mathbf{p}$ \\
\hline OWAS (1977) & $\mathrm{E}$ & C.V.D. & \multirow{2}{*}{0,3257} \\
\hline Rodgers (1992) & $\mathrm{J}$ & C.V.D. & \\
\hline Rodgers (1992) & $\mathrm{H}$ & C.V.C. & \multirow{2}{*}{$* 7,18 \mathrm{E}-07$} \\
\hline Malchaire (1998) & $\mathrm{O}$ & C.V.C. & \\
\hline Lifshitz \& Armstrong (1986) & A & M.S. & \multirow{6}{*}{$* 1,21 \mathrm{E}-29$} \\
\hline Keyserling et al. (1993) & $\mathrm{B}$ & M.S.Esq. & \\
\hline Keyserling et al. (1993) & $\mathrm{C}$ & M.S.Dir. & \\
\hline Couto (1998) & $\mathrm{D}$ & M.S. & \\
\hline OWAS (1977) & $\mathrm{F}$ & M.S. & \\
\hline I.E.A. (1995) & $\mathrm{Z}$ & M.S. & \\
\hline OWAS (1977) & G & M.I. & \multirow{2}{*}{$* 3,34 \mathrm{E}-12$} \\
\hline Rodgers (1992) & M & M.I. & \\
\hline Rodgers (1992) & I & O. & \multirow{3}{*}{$* 3,41 \mathrm{E}-10$} \\
\hline Malchaire (1998) & $\mathrm{P}$ & O. Dir. & \\
\hline Malchaire (1998) & Q & O. Esq. & \\
\hline Rodgers (1992) & $\mathrm{K}$ & C. & \multirow{3}{*}{$* 3,03 \mathrm{E}-05$} \\
\hline Malchaire (1998) & $\mathrm{R}$ & C. Dir. & \\
\hline Malchaire (1998) & $S$ & C. Esq. & \\
\hline Rodgers (1992) & $\mathrm{L}$ & $\mathrm{Os} / \mathrm{Ms}$ & \multirow{5}{*}{0,1347} \\
\hline Malchaire (1998) & $\mathrm{T}$ & P./M. Esq & \\
\hline Malchaire (1998) & $\mathrm{U}$ & P./M. Dir & \\
\hline Moore \& Garg (1995) & $\mathrm{V}$ & P./M. Dir & \\
\hline Moore \& Garg (1995) & $\mathrm{X}$ & P./M. Esq & \\
\hline RULA (1993) & $\mathrm{N}$ & Geral & \\
\hline TOTAL & & Todos & $* 6,28 \mathrm{E}-62$ \\
\hline
\end{tabular}


O check-list de Lifshitz e Armstrong* (1986 apud OLIVEIRA, 1998) leva em consideração as variáveis de estresse físico ou mecânico, força, postura, posto de trabalho, repetitividade e as ferramentas para os membros superiores. O check-list de Keyserling et al. (1993) acrescentou ao anterior cinco perguntas para as mãos e a avaliação dos hemicorpos (direito e esquerdo) em separado. Couto (1998) adicionou ao anterior alguns critérios relacionados à organização do trabalho e a sua aplicação para os membros superiores. Estas análises são superficiais pois não determinam a intensidade dos fatores, apenas identificam a presença ou não do mesmo.

A $2^{\mathrm{a}}$ Etapa do Método de Malchaire (1998) foi o aprimoramento do método do check-list Keyserling et al. (1993), acrescentando a avaliação da zona corporal de maior risco e a intensidade do fator $(0=$ nunca, $1=$ às vezes etc.). No entanto, existe uma distribuição desproporcional de perguntas para as diferentes zonas corporais, demonstradas na Tabela 4. Isto ocorreu porque o instrumento de Keyserling et al. (1993) é utilizado para a avaliação das extremidades dos membros superiores (mãos) e, portanto, enfatizando estas áreas. Como a adaptação do instrumento de Malchaire manteve a especificidade para as mãos, continua havendo uma ênfase maior na avaliação destes segmentos corporais. Um aspecto positivo do método de Malchaire é ele salientar a Zona Corporal de maior risco, o que facilita a priorização de ações em uma intervenção ergonômica. intensidade do esforço físico leva em consideração: o nível do esforço (\% de músculos envolvidos), o tempo de duração e a frequiência deste esforço, ou seja, basicamente fatores biomecânicos, no entanto não observa as compressões mecânicas, vibração e os fatores organizacionais. O método não compreende as avaliações dos hemicorpos direito e esquerdo, a repetitividade (direcionada aos punhos/mãos), avaliação ambiental, vibração, compressões mecânicas e a avaliação dos fatores de risco organizacionais. Outra limitação do instrumento é observar a freqüência e a duração da intensidade do esforço físico com valores iguais para todas os segmentos corporais, o que não é apropriado porque cada segmento importa em esforços diferentes. Apesar das limitações do método, sugere-se a aplicação deste para selecionar os grupos musculares a serem alongados durante a ginástica laboral.

O método desenvolvido por McAtamney e Corlett (1993), denominado RULA, baseia-se na observação dos fatores biomecânicos podendo ser utilizado para classificar e selecionar os postos de trabalho que apresentam a maior probabilidade da ocorrência dos D.O.R.T./L.E.R.. Porém, a análise da postura proposta pelo método, que observa a maior angulação articular na realização da tarefa, é insuficiente para uma análise. Sugere-se o acréscimo do tempo de duração da posição do segmento corporal e a aplicação do método ao hemicorpo direito e esquerdo. $\mathrm{O}$ instrumento pode ser aplicado na priorização de postos de trabalho durante uma intervenção ergonômica.

Tabela 4 - Perguntas da $2^{\text {a }}$ Etapa do método Malchaire (1998) em relação à região anatômica dos Membros Superiores

\begin{tabular}{lccc}
\hline SEGMENTO CORPORAL & PERGUNTAS & TOTAL & PERCENTUAL \\
\hline Geral (todos os segmentos do M.S.) & $2,9,10,13,14,5,16,17,20,22$ & 10 & 41,67 \\
\hline Nuca & 1 & 1 & 4,17 \\
\hline Ombros & $3,4,5$ & 3 & 12,5 \\
\hline Cotovelos & 6,23 & 2 & 8,33 \\
\hline Punhos/Mãos & $7,8,11,12,8,19,21,23$ & 8 & 33,33 \\
\hline TOTAL & & 24 & 100.00 \\
\hline
\end{tabular}

Observações: A pergunta 23 é direcionada aos Cotovelos e Punhos/Mãos, portanto foi contada duas vezes.

O método de Karhu, Kansi e Kuorinka (1977) (denominado de OWAS), propicia apenas a avaliação parcial da postura dos segmentos corporais da coluna, membros superiores e membros inferiores. Devido à avaliação ser realizada apenas no plano frontal, o método desconsidera os demais fatores de risco dos D.O.R.T.L.E.R.

O método de Rodgers (1992) avalia a intensidade do esforço físico, tendo como objeto de estudo, os vários segmentos corporais dos membros superiores. A
O método de Moore e Garg (1995) tem aplicação para as extremidades dos membros superiores, ou seja, punhos e mãos (direita e esquerda) separadamente. A análise é apropriada apenas para este segmento corporal específico, não cabendo generalizações. Salienta-se que o método relaciona alguns fatores organizacionais e biomecânicos mas não avalia os fatores ambientais, os quais devem ser observados separadamente, bem como outros fatores organizacionais (horas-extras, distribuição de tarefas, autonomia etc.).

LIFSHITZ, Y.; ARMSTRONG, J. (1986) A design checklist for control and prediction of cumulative trauma disorder in intensive manual jobs. THE HUMAN FACTORS SOCIETY ANNUAL MEETING, 30. Proceedings... pp. 837-941. 
A Minuta da I.E.A., elaborada por Colombini et al. (1999), busca a redução das "ações técnicas" realizadas pelo membro superior por um trabalhador em seu posto de trabalho. Esta redução baseia-se em fatores organizacionais, ambientais e biomecânicos. As ações técnicas analisam o movimento como um todo, e apresenta uma classificação geral, não especificando a região corporal que apresenta o maior risco. O método leva em consideração os fatores de risco biomecânicos específicos para cada segmento articular. No entanto, resultados do Índice de Exposição dos Membros Superiores "sem risco" da ocorrência dos D.O.R.T./ L.E.R., não significa que uma das articulações não apresente o risco da ocorrência dos distúrbios, pois as ações técnicas representam a média dos resultados globais para a força, postura e os movimentos articulares extremos. Se estes fatores estiverem presentes em um mesmo segmento articular, ocorrerá o risco do surgimento dos distúrbios, mas o IEMS estará adequado. O método não leva em consideração a avaliação da coluna vertebral, principalmente o segmento cervical, o qual está diretamente relacionado às ações biomecânicas dos membros superiores. Apesar das limitações do instrumento que está ainda em desenvolvimento, sugere-se a aplicação do método para a seleção dos postos de trabalho para a intervenção ergonômica, rotação de tarefas que apresentam um IEMS alto com uma que apresente valor baixo e comprovação de melhorias nos postos de trabalho.

A maioria dos instrumentos restringe a análise de variáveis biomecânicas, desconsiderando a organização do trabalho e problemas psicossociais como fator de risco para a ocorrência dos D.O.R.T./L.E.R., sendo estes fatores imprescindíveis no levantamento ergonômico (MACIEL, 1995; LUDUVIG, 1998; LIMA e ARAUJO, 1998; OLIVEIRA et al., 1998; SIGNORI et al., 1999). Salienta-se que o indivíduo pode estar desenvolvendo sua tarefa em posto de trabalho com um dimensionamento biomecânico adequado, mas caso a organização do trabalho não contemple as pausas, a rotatividade de tarefas etc., o trabalhador apresentará fadiga e, conseqüentemente, estará predisposto ao risco dos D.O.R.T./L.E.R..

A avaliação dos fatores organizacionais é apresentada pelos métodos de Couto (1998), Moore e Garg (1995) e Colombini et al. (1999). Porém, a análise da organização do trabalho não será observada detalhadamente por meio de um Check-List ou um instrumento que obedeça a uma sequiência estruturada de perguntas. Estes instrumentos objetivam uma triagem rápida dos postos de trabalho e as relações intrínsecas entre empregado e empregador só podem ser observadas no decorrer de determinado tempo. O trabalhador não relatará as suas frustrações ou angústias provenientes da organização do trabalho a um "estranho" (inclusive o ergonomista), temendo punições de seus supervisores. Portanto, a avaliação da organização do trabalho exige algum tempo para que o trabalhador venha a confiar e confidenciar ao ergonomista os problemas relacionados à organização do trabalho, sendo que esta é a principal fonte dos problemas psicossociais (MACIEL, 1995). Sugere-se que a avaliação deste fator de risco deva ser realizada em separado e construída continuadamente.

Os instrumentos devem rever a terminologia de força, pois o conceito de força é a capacidade de um grupo muscular específico exercer tensão contra uma resistência num esforço máximo (FOX et al., 1991; American College of Sports Medice, 1996; SANTARÉM, 1999). O mais apropriado é o termo de potência muscular. Este termo pode estar relacionado à capacidade de realizar trabalho de baixa intensidade por um longo período de tempo chamado de endurance muscular (exemplo: digitação) ou a capacidade de realizar trabalho muscular instantâneo (como levantar caixas) ou a intensidade do esforço, devido a estas variáveis físicas estarem relacionadas à capacidade de gerar energia na unidade de tempo.

Os conceitos de massa (<2kg: baixo risco; 2 a $4 \mathrm{~kg}$ : risco moderado etc.) relacionado como um risco de ocorrer os D.O.R.T./L.E.R. é limitado, pois o risco vai depender de outras variáveis como a aceleração, o tempo e o deslocamento (amplitude de movimento articular) desta massa (2 ou $4 \mathrm{~kg}$ ).

A descrição de uma massa de $2 \mathrm{~kg}$ pode ser um fator de risco pequeno, moderado ou alto dependendo do tipo de contração muscular, do tempo, da distância (amplitude de movimento articular), da velocidade (distância / tempo) em que for realizada a atividade. Movimentar uma peça de massa de $2 \mathrm{~kg}$, para adicionar outros componentes tendo uma base de apoio (uma mesa, por exemplo) é diferente do que utilizar uma marreta para fixar pedras na rua (calceteiro). Na montagem industrial, a qual compreende esta pesquisa, dentro do ciclo de trabalho existem pequenas pausas onde a peça é apoiada na mesa. No entanto, por exemplo, o calceteiro apresenta uma agarra manual fixa, sem pausas, onde transporta a ferramenta (marreta) constantemente próximo ao limite da fadiga muscular.

A análise ergonômica ficaria mais fidedigna, facilitando a intervenção, bem como a comprovação dos resultados, caso os protocolos designassem o segmento corporal que estaria predisposto aos riscos de desenvolverem os D.O.R.T./L.E.R.. Excetua-se desta consideração os protocolos de Rodgers (1992) e Malchaire (1998) que indicam o segmento corporal.

Observa-se que os protocolos proporcionam uma variabilidade de resultados intergrupo. No mesmo 
posto de trabalho e segmento corporal, pode-se encontrar resultados que indiquem a ausência ou a presença do risco da ocorrência dos D.O.R.T./L.E.R., dependendo do instrumento utilizado. Este fato pode levar a uma interpretação errada do ergonomista em relação ao posto de trabalho em estudo, podendo induzir a uma triagem inapropriada dos postos de trabalho e, consequientemente, a uma intervenção ergonômica desnecessária.

$\mathrm{Na}$ análise dos resultados intragrupo, observada pela análise das variâncias, descritas na Tabelas 5, observase que os protocolos apresentam uma variação pequena para os instrumentos OWAS (1977) de segmento dos membros inferiores, I.E.A. (COLOMBINI, et al., 1999) para os membros superiores e RULA (McATAMNEY e CORLETT, 1993) para a análise geral do posto. Apresentaram uma variação moderada os instrumentos de Lifshitz e Armstrong* (1986 apud OLIVEIRA, 1998) para os membros superiores, Keyserling et al. (1993) para as extremidades dos membros superiores (esquerda e direita), Couto (1998) para os membros superiores, OWAS (1977) para o braço, Rodgers (1992) para os membros inferiores e Malchaire (1998) para a coluna cervical e ombros (direito e esquerdo). Já os protocolos OWAS (1977) para a coluna dorsal; Rodgers (1992) para a coluna cervical, dorsal, ombro, cotovelos e punhos/mãos; Malchaire (1998) para os cotovelos e punhos/mãos e o de Moore \& Garg (1995) para os membros superiores, apresentaram uma grande variância de resultados intragrupo.

As variações intragrupo se devem à subjetividade na interpretação dos fatores de risco para os D.O.R.T./ L.E.R. estabelecidos pelos protocolos, salientando-se que a variação das médias individuais é pequena, o que representa que a amostra é homogênea.

A utilização destes instrumentos para a comprovação das melhorias propiciadas pela intervenção ergonômica apresentará relevância apenas em alguns fatores de risco específicos analisados pelos protocolos. Com esta finalidade, destaca-se o método I.E.A., que se encontra em desenvolvimento. Ele objetiva determinar o número máximo de ações técnicas que o trabalhador pode realizar num posto de trabalho. Melhoradas estas condições pelos ergonomistas, o número de ações técnicas pode aumentar até o limite máximo de 30/min, comprovando, assim, as melhorias. Para os demais instrumentos, deve-se observar quais fatores de risco para o desenvolvimento dos D.O.R.T./L.E.R. que se deseja avaliar e escolher o protocolo mais apropriado.
Devido à variação dos resultados intra e intergrupo, os protocolos de avaliação simplificada dos riscos dos D.O.R.T./L.E.R. são pouco consistentes, não propiciando resultados fidedignos. Estes métodos servem para analisar alguns fatores de risco específicos. É preciso entender que uma intervenção ergonômica adequada é um processo contínuo e não uma solução imediata. Os instrumentos podem observar se algumas variáveis estão adequadas ao ser humano nos postos de trabalho. Melhorando estas variáveis, diminui-se a probabilidade da ocorrência dos D.O.R.T./L.E.R., mas não se impede que esta venha a se desenvolver, devido à complexidade de fatores de risco envolvidos.

\section{Conclusões}

Os nove instrumentos analisados nesta pesquisa buscam uma rápida e prática avaliação e classificação do risco da ocorrência dos D.O.R.T./L.E.R., sem a necessidade de equipamentos sofisticados. No entanto, estes devem ser utilizados apenas em postos de trabalho onde a postura corporal geral seja preponderantemente estática (em pé, sentado e/ ou a alternância destas), pois a natureza dos protocolos não permite sua aplicação a postos de trabalho que exigem o deslocamento do trabalhador.

Observou-se que há uma heterogeneidade dos resultados entre os nove instrumentos utilizados na pesquisa, sendo que no mesmo posto de trabalho o risco foi classificado como baixo, moderado ou alto, dependendo do instrumento empregado. Esta variação intergrupo se deve aos critérios de avaliação dos fatores de riscos utilizados apresentarem valores diferentes para a mesma variável, remetendo à necessidade de estudos para a padronização dos mesmos.

Os resultados intragrupo variam devido à subjetividade da interpretação dos fatores de risco para os D.O.R.T./L.E.R. estabelecidos pelos instrumentos analisados. Esta subjetividade não leva ao consenso dos resultados da análise. Salienta-se que os instrumentos que apresentam uma menor variabilidade são: OWAS (1977) na análise dos segmentos dos membros inferiores, I.E.A. (1999) na análise dos membros superiores e RULA (1993) na análise geral do posto.

A variabilidade dos resultados da classificação do risco da ocorrência dos D.O.R.T./L.E.R., intra e intergrupo induzem os ergonomistas a erros na seleção e na intervenção ergonômica dos postos de

\footnotetext{
* LIFSHITZ, Y.; ARMSTRONG, J. (1986) A design checklist for control and prediction of cumulative trauma disorder in intensive manual jobs. THE HUMAN FACTORS SOCIETY ANNUAL MEETING, 30. Proceedings... pp. 837-941.
} 
trabalho. Os instrumentos de avaliação do risco da ocorrência dos D.O.R.T./L.E.R., utilizados pelos ergonomistas, não contemplam uma intervenção macroergonômica (não consideram fatores psíquicos e sociais e a maioria, fatores organizacionais importantes) mas servem para analisar fatores de risco específicos nos postos de trabalho. Os fatores analisados são preponderantemente de natureza biomecânica, havendo a necessidade da utilização de mais de um instrumento de avaliação, para garantir maior consistência da análise e, conseqüentemente, melhorar a confiabilidade e a fidedignidade dos resultados que uma intervenção ergonômica necessita.

\section{Referências}

AMERICAN COLLEGEOF SPORTS MEDICINE. Manual Testes de Esforço e Prescrição de Exercício. $4^{\circ}$ ed. Rio de Janeiro, RJ.: Livraria e Editora Revinter Ltda., 1996.

BRASIL NR - 17 Ergonomia. Redação dada pela Portaria $n^{\circ}$. 3.751, de 23 de novembro de 1990. In: MANUAIS DE LEGISLAÇÃO ATLAS - Segurança e medicina do trabalho. $43^{\circ}$ ed. São Paulo,SP.: Atlas S.A., (16): $217-220,1999$.

BRASIL. Lei no 8.213 de 24 de junho de 1992; Decreto $\mathrm{n}^{\circ} 2172$, de 05 de março de 1997. Aprova a Norma Técnica sobre os Distúrbios Osteoarticulares Relacionados ao Trabalho - DORT. Diário Oficial da União, Brasília, Ordem de Serviço nº 606, Of. n 308/98, p. 26 38, 5 de agosto de 1998. Seção I.

BRASIL. Portaria 4062 de 06 de julho de 1987, Aprova a norma técnica sobre as L.E.R. do Instituto Nacional de Seguridade Social.

COLOMBINI, D.; OCCHIPINTI, E.; DELLEMAN, N.; FALLENTIN, N.; KILBOM, A. \& GRIECO, A.

Exposure assessment of upper limb repetitive movements: a consensus document. Developed by the Technical Committee on Musculoskeletal Disorders of International Ergonomics Association (IEA) endorsed by Commission on Occupational Health (ICOH), Milano, Italy, march, 1999.

COUTO, Hudson de Araújo Avaliação dos riscos de D.O.R.T./L.E.R. In: COUTO, H. A.; NICOLETTI, S. J.; LECH, O. e Colaboradores. "Como gerenciar a questão das L.E.R./D.O.R.T.: Lesões por Esforços repetitivos / Distúrbios Osteomusculares relacionados ao Trabalho" $1^{\circ}$ Ed. Belo Horizonte,MG.: Ergo, 1998.

DIMON, A. History 'repeats' itself. Journal: Benefits Canadá, 21 (5): 15, Maio de 1997.

FOX, E. L.; BOWERS, R. W.; FOSS, M. L. Bases fisiológicas da educação física e dos desportos. $4^{\circ}$ edição, Rio de Janeiro,RJ.: Guanabara Koogan S.A., 1991
KAPANDJI, I. A. Fisiologia articular - Esquemas e comentários da mecânica humana $5^{\circ}$ ed., São Paulo, SP.: Manole Ltda, Vol. 1, 2 e 3, 1990.

KARHU, O.; KANSI, P.; KUORINKA, I. I. Correcting working postures in industry: A practical method for analysis. Journal of Applied Ergonomics, Helsinki, Finland, 8 (4): 199-201, 1977.

KEYSERLING, W. M.; STETSOND. S.; SILVERSTEIN B. A.; BROUWER, M. L. "A cumulative trauma disorders". Journal of Ergonomics. 36 (7): 807-831, 1993.

LIMA, M. E. A.; ARAÚJO, J. N. G.; LIMA, F. P. A.. L.E.R. Dimensões Ergonômicas e Psicossociais. $1^{\circ} \mathrm{ed}$. Belo Horizonte, MG.: Health, 1998.

LUDUVIG, M. M. "Doenças ocupacionais fuja desse castigo”. Revista Saúde é Vital, Editora Azul, (174):4759, 1998.

MACIEL, R. H. Ergonomia e lesões por esforços repetitivos (LER). In: CODO, W.; ALMEIDA, M. L.E.R. Lesões por Esforços Repetitivos $4^{\circ}$ ed., Petrópolis, RJ.: Vozes, 1995.

MALCHAIRE, J. Estrategia de Prevencioan - Lesiones de membros superiores por trauma acumulativo. $2^{\circ}$ ed. Bélgica: INCRT, 1998.

McATAMNEY, L.; CORLETT, N. RULA: a survey method for the investigation of work-related upper limb disorders. Journal Applied Ergonomics, Butterwoth-Heinemann Ltda. 24(2):91-99, 1993.

MOORE, J. S.; GARG, A. The strain index: A proposed method to analyze jobs for risk of distal upper extremity disorders. American Industrial Hygiene Association Journal, 56:443-458, 1995.

NIOSH-NATIONALINSTITUTEFOR OCCUPATIONAL SAFETY AND HEALTH Musculoskeletal disordens NIOSH research projets. Janeiro de 1997 [online] Disponível na Internet via www.cdc.gov/niosh/homepage.html. Arquivo capturado em 15 de dezembro de 1998.

OLIVEIRA, C. R. e Colaboradores. Manual prático de L.E.R. -Lesões por Esforços Repetitivos. 1 $^{\mathrm{a}}$. ed. Belo Horizonte, MG.: Livraria e Editora Saúde Ltda - Health, 1998.

RODGERS, S. H. A functional job analysis technique. Occup. Med.: State of the Art Reviews. 7(4):679-711, 1992.

SANTAREM, José Maria. Treinamento de Força e Potência In: GHORAYEB, Nabil; BARROS, Turíbio. O exercício - Preparação fisiológica, avaliação médica / aspectos especiais e preventivos. $1^{\circ} \mathrm{ed}$, Atheneu, São Paulo, 1999. Cap. 4 p. 35-50. 
Signori, L. U.; Guimarães, L. B. M.; Sampedro, R. M. F. -Análise dos instrumentos utilizados para a avaliação... 61

SIGNORI,L.U.; MARTINS, M. A.P.; ANTONELLO, N.; GODOY, L. P. Gerenciamento das LER/DORT através da organização do trabalho In: ANAIS XIX ENEGEP_-

Encontro Nacional de Engenharia de Produção V ICIE International Congress of Industrial Engineering III

Profundão - Encontro de Engenheiros de Produção da UFRJ. Rio de Janeiro, novembro de 1999.
SILVERSTEIN, B. A.; FINE, L. J.; ARMSTRONG T. J. Hand wrist cumulative trauma disorders in industry. British Journal of Industrial Medicine. 43:779-784, Michigan, U.S.A. 26 January, 1986.

Submetido em abril/2002

Aprovado em julho/2002 


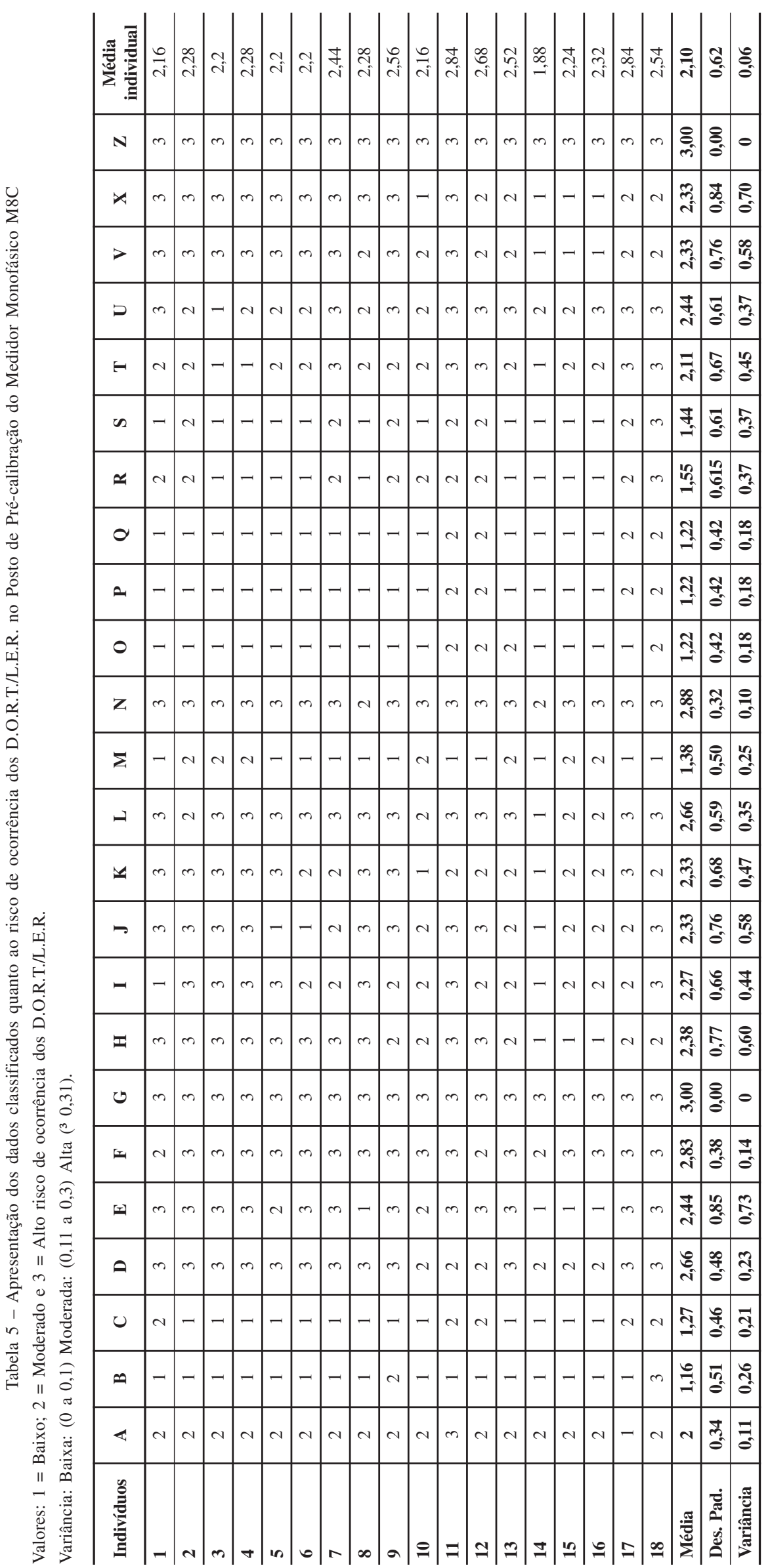

